\title{
PROBLEMAS DERIVADOS DE LA RELACIÓN JURÍDICA ENTRE PEQUEÑO EMPRESARIO TURÍSTICO Y WEB 2.0
}

\author{
PROBLEMS DERIVED FROM THE LEGAL RELATIONSHIP \\ BETWEEN SMALL TOURIST ENTREPRENEUR AND WEB 2.0 \\ Dra. FRANCISCA Ma ROSSELLÓ RUBERT ${ }^{1}$ \\ Profesora Ayudante de Derecho Mercantil \\ Departamento de Derecho Privado - Área de Derecho Mercantil \\ Facultad de Derecho \\ Universitat de les Illes Balears
}

\begin{abstract}
Resumen
El auge de las Webs 2.0 y del software como servicio en el mercado del sector turístico ha supuesto un punto de no retorno en cuanto a la promoción y a la reputación en línea de establecimientos hosteleros y de oferta complementaria. Por una parte, el pequeño empresario deberá atender a las condiciones contractuales que suscribe como usuario de tales plataformas. En estos casos, es importante conocer aspectos como las políticas de uso adecuado, la protección de contenidos digitales con propiedad intelectual o la distribución de responsabilidades entre proveedor de servicios digitales y empresario suministrado. Pero ¿qué sucede en aquellos casos en los cuales el empresario no ha dado su consentimiento para que su establecimiento figure en una determinada Web 2.0?

Este trabajo propone soluciones en aquellos casos en los que media un contrato entre plataformas Web 2.0 (u otros servicios digitales similares), como en los casos en los que no existe contrato que vincule al empresario con el proveedor y, sin embargo, el empresario ve mermada su posibilidad de decisión sobre la reputación y presencia digital en la Web 2.0.
\end{abstract}

\section{Palabras clave}

Plataformas digitales, comparadores, opiniones de usuarios, contratos online, reputación online

\section{Abstract}

The rise of Web 2.0 and software as a service in the tourism sector market has meant a point of no return in terms of the promotion and online reputation of hotel establishments and complementary offerings. On the one hand, the small business owner must comply with the contractual conditions that he subscribes as a user of such platforms. In these cases, it is important to know aspects such as adequate use policies, the protection of digital content with

${ }^{1}$ francisca.rossello@uib.es 
intellectual property or the distribution ofres ponsibilities between the digital service provider and the entrepreneur supplied. But what happens in those cases in which the employer has not given his consent for his establishment to appear in a certain Web 2.0?

This work proposes solutions in those cases in which there is a contract between Web 2.0 platforms (or other similar digital services), such as in cases where there is no contract linking the employer with the provider and yet the employerse es reduced its possibility of decision on the reputation and digital presence in Web 2.0.

\section{Keywords}

Digital platforms, user reviews, small business, online contracts, online reputation,

Fecha de recepción: 17 de octubre de 2018 Fecha de aprobación: 28 de noviembre de 2018

\section{INTRODUCCIÓN ${ }^{2}$}

Algunas plataformas web soportadas por tecnología cloud han supuesto un enorme impacto en el sector turístico, cambiando el "ecosistema económico" y dando mucha más participación al usuario y a su valoración de la experiencia sobre el producto o servicio turístico disfrutado ${ }^{3}$. Estas Webs 2.0 permiten al internauta crear y compartir contenidos a través de interfaces sencillas, y no siempre es fácil distinguirlas del modelo comercial del software como servicio gratuito ${ }^{4}$.

Existen, en la actualidad, múltiples Webs 2.0 de diferentes categorías y con distintas funcionalidades: buscadores, comparadores, foros para compartir y valorar experiencias, etc ${ }^{5}$. Algunas de estas plataformas, además, posicionan a las empresas y establecimientos turísticos de acuerdo con las valoraciones de los usuarios $\mathrm{u}$ otros

2Esta investigación se ha elaborado gracias al disfrute de la ayuda para formación de personal investigador predoctoral concedida por la Conselleríad'Innovació, Recerca $i$ Turisme del Gobierno de las Islas Baleares y del Fondo Social Europeo, en el marco del Programa Operativo FSE 2014-2020. Igualmente, el presente trabajo se ha realizado en el marco de los Proyectos "Turismo y nuevas tecnologías; en especial, el régimen jurídico de las denominadas centrales electrónicas de reservas turísticas" (DER2012-32063), y "Big data, Cloud Computing y otros nuevos retos jurídicos planteados por las tecnologías emergentes; en particular, su incidencia en el sector turístico" (DER2015-63595), ambos bajo la dirección de la Catedrática Apol·lònia Martínez Nadal como investigadora principal, financiados por la Dirección General de Investigación, del Ministerio de Economía y Competitividad y desarrollados en la Universitat de les Illes Balears.

3 Algunos portales, como TripAdvisor o Booking, han cambiado completamente el "ecosistema económico" del sector turístico, y dan al usuario una mayor participación, al permitirle valorar la experiencia y el producto final. PALOS SÁNCHEZ, Pedro; AGUAYO CAMACHO, Mariano; Los ecosistemas de turismo..., op. cit., pág. 136.

4Sobre una distinción entre el Cloud Computing y la Web 2.0, nos remitimos a: ROSSELLÓ RUBERT, Francisca M., Cloud Computing. Régimen jurídico para empresarios, Navarra, 2017, págs. 49 y ss.

5 Como ejemplo de una Web 2.0 que integra múltiples funcionalidades, podemos mencionar Minube. Esta plataforma se describe como "una comunidad de viajeros" que integra un comparador de precios de vuelos, alquileres de coches y hoteles, una red social que permite crear un perfil con gustos y preferencias y compartir las experiencias de viajes con otros usuarios. También permite a las empresas mejorar su posicionamiento. 
mecanismos, y, a su vez, permiten realizar reservas o adquirir servicios turísticos de las mismas.

En este trabajo comentaremos algunos aspectos jurídicos en el ámbito de las plataformas Web 2.0, y su relevancia para el pequeño empresario del sector turístico, así como la problemática más frecuente. Antes de adentrarnos en estas cuestiones consideramos necesario, sin embargo, delimitar el concepto de Web 2.0.

\section{CONCEPTO DE WEB 2.0}

La evolución de las páginas web ha permitido el disfrute de funcionalidades de software diverso que se prestan a través del acceso a sitios web mediante un navegador o servidor web. Si la llamada Web 1.0 únicamente permitía al internauta leer contenidos publicados en el sitio web, este concepto se superó con la Web 2.0, al aprovecharse las ventajas de Internet para desarrollar un mecanismo de colaboración entre usuarios y sitios web, creando contenidos interactivos ${ }^{6}$.

Los caracteres que definen a la Web 2.0 son su asimilación a un servicio que permite economías de escala, en contraposición con el software empaquetado tradicional; que se enriquece cuanto más usuarios lo utilizan; que confía en los usuarios como desarrolladores de contenidos; que facilita su uso autónomo por el cliente y sin necesidad de ser auxiliado por el proveedor o de necesitar elevados conocimientos técnicos; y que utiliza interfaces sencillas para conseguir lo anterior ${ }^{7}$.

En nuestra opinión, y coincidiendo con algunos autores, la Web 2.0 primitiva es uno de los múltiples elementos tecnológicos que ayudaron a crear los cimientos de los actuales servicios de computación en la nube ${ }^{8}$. Asimismo, la fusión de ambas tecnologías y la evolución de la Web 2.0 tiene como resultado que las funcionalidades de algunas Webs 2.0 sean susceptibles de ser prestadas a modo de servicio digital, con lo cual resulta dificultoso en ocasiones deslindar donde acaba la Web 2.0 y donde empieza el software como servicio característico del Cloud Computing, especialmente respecto de aquellas que no exigen al usuario una contraprestación monetaria9 ${ }^{9}$ De entre estas funcionalidades,

6Ejemplos conocidos del concepto de Web 2.0 previo a la aparición de la computación en la nube podrían ser Wikipedia, Youtube o TripAdvisor.

7O'REILLY, Tom; "Whatis Web 2.0: DesignPatterns and Business Modelsforthenextgenerationof Software", Communications\&Strategies, núm. 65, 2007, pág. 37.

8 "We can tracktherootsofcloudscomputingbyobservingtheadvancementofseveraltechnologies, especially in hardware (virtualization, multi-core chips), Internet technologies (Web services, service-orientedarchitectures, Web 2.0), distributedcomputing (clusters, grids), and systemsmanagement (autonomiccomputing, data center automation)". VOORSLUYS, William; BROBERG, James; BUYYA, Rajkumar, "Introductionto Cloud Computing", Cloud Computing Principles and Paradigms(Coord. RajkumarBuyya, James Broberg, AndrzejGoscinski), $1^{\text {a }}$ edición, New Jersey, 2011, pág. 5.

9La actual convergencia de la Web 2.0 con la tecnología del Cloud Computing supone que, especialmente en los softwares como servicio, sea complicado en ocasiones distinguir entre ambas, puesto que ambas 
destacamos las siguientes categorías, a título de ejemplo y como lista abierta: comunicación social ${ }^{10}$, compartición de información ${ }^{11}$, almacenamiento de contenidos ${ }^{12}$, edición colaborativa ${ }^{13}$, marcado y organización de enlaces y archivos en línea (también llamados marcadores sociales) ${ }^{14}$; etc.

Ha de mencionarse que la Propuesta de Directiva del Parlamento Europeo y del Consejo relativa a determinados aspectos de los contratos de suministro de contenidos digitales (COM (2015) 634 final), asimilaba en su versión inicial el suministro de contenidos digitales a la puesta a disposición del cliente de tales contenidos digitales a través de Internet, tal y como tiene lugar en muchas Webs 2.0: "«suministro»: hecho de facilitar el acceso a contenidos digitales o poner a disposición los contenidos digitales"15. En posteriores revisiones de la Propuesta,se definen servicios digitales como aquellos servicios que permiten al consumidor crear, procesar, almacenar o acceder a datos en formato digital, o bien aquellos servicios que permiten la compartición u otras interacciones con datos en formato digital subidos o compartidos por el consumidor $u$ otros usuarios del servicio ${ }^{16}$.

tecnologías se fusionan en la práctica. Al respecto, nos encontramos con literatura contradictoria respecto a tal diferenciación, especialmente en lo referente a redes sociales y servicios de correo electrónico. Por ejemplo, autores como Simon Bradshaw, Ian Walden y Christopher Millard, de la Queen Mary Universityof London, o Michael Gordon y KathreenMarchesini, de la Universityof North Carolina, consideran a las redes sociales y al correo electrónico como software como servicio, y, por tanto, servicios de computación en la nube. Del mismo modo, la norma ISO-IEC 17788:2014 considera las comunicaciones como servicio (es decir, correo electrónico y redes sociales) una "categoría de serviciocloud en la cual la capacidad provista al cliente es interacción y colaboración en tiempo real" (traducción propia). Sin embargo, Michael Armbrust y Armando Fox, de la Universidad de Berkeley, consideran que las redes sociales son servicios diferentes a la computación en la nube, aunque se sirven de ella como tecnología de soporte. BRADSHAW, Simon; MILLARD, Christopher; WALDEN, IAN; "Standard contractsfor Cloud Computing Services", Cloud Computing Law, 1ª edición, Oxford, 2013, pág. 41. GORDON, Michael; MARCHESINI, Kathryn; Examplesof Cloud Computing Services [en línea], 2010. Disponible en: <https://www.unc.edu/courses/2010spring/law/357c/001/cloudcomputing/examples.html<. [Fecha de consulta: 21 de septiembre de 2018]. ARMBRUST, Michael; FOX, Armando [et al]; "AbovetheClouds: a Berckeley ...", op. cit, pág 8. Para un estudio más detallado sobre los aspectos jurídicos de los contratos de Cloud Computing y su diferenciación con la Web 2.0, nos remitimos a: ROSSELLÓ RUBERT, Francisca M., Cloud Computing. Régimen jurídico para empresarios, Navarra, 2017, 444 págs.

10Por ejemplo, blogs de participación abierta (es decir, no solo de lectura) y redes sociales como Facebook, Twitter o Linkedin, o servicios de correo electrónico como Gmail o Hotmail.

11 Por ejemplo, Youtube.

12Por ejemplo, Box o Dropbox.

13Por ejemplo, Google Docs, Microsof Office 365 o Zoho.

14Ejemplos de marcadores sociales son Delicious o Evernote. Permiten organizar y almacenar por categorías o etiquetas enlaces web y poderlos recuperar posteriormente.

15Cabe destacar que la definición de "suministro", inicialmente recogido en el documento COM (2015) 634 final, desapareció en posteriores revisiones de la mencionada Propuesta de Directiva concretamente en su versión 2017 9901/17 ADD 1.

16Traducción propia de la versión original en inglés de la Propuesta 2017 9901/17 ADD: “1a. 'digital service' means: (a) a serviceallowingtheconsumerthecreation, processingorstorageof, oraccessto, data in digital form (...); or(b) a serviceallowingthesharingoforanyotherinteractionwith data in digital formuploadedorcreatedbytheconsumer and otherusersofthatservice". La versión anterior de la Propuesta COM 
Asimismo, puede entenderse que esta Propuesta de Directiva acoge, dentro del concepto de "contenidos digitales", múltiples contenidos y funcionalidades digitales ${ }^{17}$.

Todos los servicios de suministro de contenidos digitales son susceptibles de ser suscritos por pequeños empresarios, quienes se verán afectados por muchas de las cuestiones jurídicas y aspectos contractuales relacionados con el almacenamiento remoto y la recuperación de datos, la deslocalización de la información, la protección de datos personales y la distribución de responsabilidades entre usuario y proveedor. Sin embargo, los pequeños empresariosquedarían fuera de la protección de la Propuesta de Directiva de suministro de contenidos digitales, una vez que esta llegue a aprobarse, puesto que esta regulación se destina a proteger a los usuarios consumidores.

En este trabajo, dedicado especialmente al pequeño empresario del sector turístico, nos referiremos a la problemática jurídica de Webs 2.0 que permiten la valoración y comparación de servicios turísticos.

\section{ALGUNOS ASPECTOS JURÍDICOS DE LOS CONTRATOS PREDISPUESTOS POR COMPARADORES DE SERVICIOS TURÍSTICOS}

(2015) 634 final recogía una definición más completa, en la cual se detallaban tipologías de contenidos digitales: "art. 2.1: «contenido digital»: a) datos producidos y suministrados en formato digital, por ejemplo vídeo, audio, aplicaciones, juegos digitales y otro tipo de software, b) servicio que permite la creación, el tratamiento o el almacenamiento de los datos en formato digital, cuando dichos datos sean facilitados por el consumidor, y c) servicio que permite compartir y cualquier otro tipo de interacción con datos en formato digital facilitados por otros usuarios del servicio". (Original en castellano). En el texto de la Propuesta de 2017 se sugiere que tal enumeración ejemplificativa de datos digitales se recoja en la Exposición de Motivos: "Therecitalsshouldlistexamplesof digital content (movies, music files, games, e-books orother e-publications, which can be eitherdownloadedorstreamed online) whichcould be wordedalongthefollowinglines: "ThisDirectiveshouldaddressproblemsacrossdifferentcategoriesof digital content, digital services and itssupply. In ordertocaterforfasttechnologicaldevelopments and tomaintainthefuture-proofnatureofthenotionof digital content, thisDirectiveshouldcover, amongothers, movies, music files, games, e-books orother e-publications, and also digital serviceswhichallowthecreation, processingorstorageof data including software-as-a-servicesuch as video and audio sharing and other file hosting, wordprocessingorgamesoffered in thecloudcomputingenvironment and social media. Whilethere are numerouswaysfor digital contentto be supplied, such as transmissionon a tangible medium, downloadingbyconsumersontheirdevices, web-streaming, allowingaccesstostoragecapabilitiesof digital contentoraccesstothe use of social media, thisDirectiveshouldapplytoall digital content and digital servicesindependentlyofthemediumusedforthetransmissionortheprovisionofaccesstoit. However, thisDirectiveshouldnotapplytotheprovisionofaccesstothe internet"), así como la ejemplificación de servicios digitales: "Therecitalsshouldalsolistexamplesof 'digital services'in therecitals: software-as-a-servicesuch as wordprocessing, video and audio file editing, games and anyother software offered in thecloudcomputingenvironment, sharing and other file hosting (seeproposed recital language in thepreviousfootnote)".

17Los contratos de computación en la nube jugaron un papel particularmente importante en la identificación de los problemas contractuales relevantes para la mencionada Propuesta de Directiva. Estas cuestiones, que fueron debatidas en profundidad por el Grupo de Expertos en Contratos de la Cloud Computing Strategy, estaban relacionadas con la calidad, la responsabilidad o la modificación de los contratos. Así se reconoce en los documentos de trabajo de la propia Propuesta de Directiva, concretamente al explicarse la obtención de asesoramiento técnico para su redacción. 
Las Webs 2.0 relacionadas con el sector turístico tienen distintas finalidades, dependiendo del servicio, y generalmente se utilizan para reunir información sobre viajes, realizar reservas, comparar precios, publicar opiniones y compartir experiencias.

Los proveedores de Webs 2.0 generalmente sujetan el uso de sus plataformas a la aceptación de ciertas condiciones contractuales. En ocasiones se entienden aceptadas por el mero uso del sitio web, y en otras se advierte al internauta que no está autorizado a abrirse una cuenta de usuario y utilizar estas funcionalidades si no está de acuerdo con todas las condiciones ${ }^{18}$. Como se deduce de lo anterior, el usuario de estas Webs 2.0, ya sea particular o pequeño empresario, no dispone de capacidad de negociación alguna, con lo cual, si desea hacer un uso interactivo de esta plataforma, deberá aceptar las condiciones predispuestas por el proveedor. Además, puesto que muchas de estas plataformas se prestan sin exigencia de remuneración económica al usuario, este no siempre se detendrá a examinar detalladamente el contenido de esas condiciones contractuales ${ }^{19}$.

Como también sucede en la mayoría de las condiciones de servicios prestados a través de Internet, el proveedor de la plataforma web se reserva la facultad de modificar los términos legales, así como los contenidos o las funcionalidades de la plataforma, en cualquier momento y sin necesidad de previo aviso ${ }^{20}$. Por ello es recomendable que el usuario, especialmente si se trata de un pequeño empresario, revise eventuales modificaciones en la versión del acuerdo legal antes de proceder a prestar su consentimiento, y que el proveedor, por su parte, destaque la fecha de la última modificación y, a ser posible, averigüe los contenidos que han sido modificados.

A continuación, nos centraremos en algunos de los aspectos contractuales comunes entre la contratación de servicios digitales, y en otros aspectos que son específicos de estas plataformas.

\subsection{Políticas de uso adecuado y veracidad de los contenidos compartidos por el usuario}

Los usuarios de Webs 2.0 se comprometen a usar el servicio de manera ética y leal.

18 A modo de ejemplo, véanse los términos de servicio de las plataformas TripAdvisor, Minube yBooking. Disponibles respectivamente en: <https://tripadvisor.mediaroom.com/ES-terms-of-use>, < https://www.minube.com/politica-privacidad> y <http://www.booking.com/content/terms.es.html >. [Fecha de consulta: 24 de septiembre de 2018].

19 Para un estudio más detallado de las contraprestaciones no dinerarias, nos remitimos a ROSSELLÓ RUBERT, Francisca María, Las contraprestaciones no dinerarias en la Propuesta de Directiva sobre suministro de contenidos digitales. En: Revista de Derecho Mercantil, núm. 303, 2017, págs. 163-190.

20 Como ejemplo, la reserva efectuada por el comparador Rastreator.com: "Rastreator.com se reserva el derecho a modificar en cualquier momento y sin necesidad de previo aviso la presentación, configuración y contenidos de la website de Rastreator.com, así como el presente "Aviso Legal”, como cualesquiera otras particularidades que acaso existieren para determinados espacios, servicios o usos. Por este motivo, Rastreator.com recomienda a los usuarios que lean detenidamente el "Aviso Legal" y "Política de Privacidad" con anterioridad a que acepten la casilla para prestar su consentimiento". Aviso legal disponible en: <http://www.rastreator.com/aviso-legal.aspx>. [Fecha de consulta:24 de septiembre de 2018]. 
Por ello, los contratos de estas plataformas incorporan las denominadas políticas de uso adecuado (PUA), un conjunto de cláusulas que prohíben ciertos usos y abusos de los contenidos y funcionalidades proporcionados por el servicio (por ejemplo, la publicación de mensajes de contenido sexual, difamatorio, ofensivo u obsceno; la publicación de datos de contacto de terceros, la difusión de spam o malware utilizando la plataforma, sobrecargar el servicio, etc. $)^{21}$.

Si bien los proveedores de estas plataformas suelen afirmar que eliminarán los contenidos de aquellos usuarios que no cumplan con estas políticas (a su entera discreción $\mathrm{y}$ sin establecerse procedimientos de réplica por parte del usuario) ${ }^{22}$, también suelen incorporar en sus condiciones una exención de responsabilidad ante terceros respecto de tales infracciones ${ }^{23}$.

21El comparador de sitios webs de reserva Trivago establece en sus términos de uso las siguientes obligaciones para los usuarios respecto del uso del servicio: "Es responsabilidad de los usuarios adquirir los derechos sobre los contenidos (texto, fotografías, opiniones, enlaces, etc.) que añadan a Trivago, lo que significa que deben asegurarse de poseer todos los derechos sobre los contenidos que publiquen en la plataforma de Trivago y de que estos contenidos no violen los derechos de terceros. Los usuarios se comprometen a no utilizar los servicios de Trivago para crear los siguientes tipos de contenidos: publicidad encubierta; contenidos no relacionados con el elemento sobre el que se está opinando; contenidos subjetivos o intencionadamente falsos; contenidos inmorales, pornográficos u ofensivos; contenidos que infrinjan los derechos de terceros y, más concretamente, los derechos de autor; contenidos que sean contrarios a la ley vigente o constitutivos de delito; contenidos infectados con virus u otros programas que puedan dañar el software o el hardware o que puedan afectar al correcto uso y/o funcionamiento de los ordenadores; encuestas o cartas en cadena; y contenidos que tengan como objetivo recoger o usar los datos personales de otros usuarios, especialmente para fines comerciales. Los usuarios se comprometen a no usar programas o funciones para la generación automática de opiniones o contenidos enTrivago". Asimismo, establece que "en caso de incumplimiento del presente contrato de términos y condiciones, Trivago se reserva el derecho a eliminar el contenido sin necesidad de declarar los motivos, a retener los pagos obtenidos en la Comunidad de Trivago y a bloquear permanentemente al miembro en cuestión. Esto no afectará al derecho a incoar una causa penal". Términos del servicio disponibles en: <https://www.trivago.es/terminos-y-condiciones>. [Fecha de consulta: 24 de septiembre de 2018].

22La plataformaTrivago afirma que "en caso de que se ponga en conocimiento de Trivago que un miembro está haciendo un uso inadecuado de su web, Trivago podrá retirar el acceso al usuario y eliminar su registro en el plazo de una semana tras recibir la información. El usuario también podrá cancelar su propio acceso y registro dentro del mismo plazo. Lo anterior no afectará al derecho a la resolución inmediata por una causa justificada". Términos del servicio disponibles en: <https://www.trivago.es/terminos-y-condiciones>. [Fecha de consulta: 24 de septiembre de 2018].

23Minube se compromete a eliminar los contenidos que infrinjan las prohibiciones establecidas por los términos de uso: "12.2. Minube se reserva el derecho de no publicar aquellos contenidos que resulten contrarios a las presentes normas, así como retirar todo artículo o comentario publicado que no se ajuste a las mismas, sin notificación de ninguna clase. Minube se reserva el derecho de cancelar el registro y entrada a la comunidad de viajeros en la medida que determinados usuarios reincidan en este tipo de conductas (...). Por ello serán eliminados de Minube los perfiles de usuarios que contengan: lenguaje o sugerencias de tipo sexual, fotografías sexualmente sugerentes, contenido difamatorio, abusivo, agresivo o violento, lenguaje ofensivo u obsceno", aunque también afirma que, si bien "periódicamente se realizan exhaustivos controles para asegurar que se cumplen las políticas y se mantiene la calidad de lo publicado", no se responsabiliza por esos contenidos: "dado que es imposible que Minube pueda controlar todos los contenidos publicados, no puede asumir la responsabilidad sobre los mismos. Cualquier usuario que proporcione información o realice actividades que contravengan la legalidad vigente asumirá la responsabilidad exclusiva de los perjuicios y consecuencias derivadas de la misma, eximiendo a Minube de cualquier responsabilidad. (...)". [Fecha de consulta: 24 de septiembre de 2018]. 
Al respecto de estas exenciones de responsabilidad, resulta altamente recomendable que el pequeño empresario sea consciente de los riesgos que pueda suponerle el uso de esa Web 2.0. Además, en nuestra opinión, el juzgador, en su caso, deberá atender a la legalidad y proporcionalidad de tales exenciones o limitaciones de responsabilidad que puedan perjudicar injustificadamente al pequeño empresario, pudiéndose realizar, en ciertas ocasiones, una interpretación extensiva de la protección establecida para el usuario consumidor, siempre y cuando el pequeño empresario se halle en una situación asimilable (imposibilidad de negociación, desequilibrio contractual, etc. $)^{24}$.

\subsection{Propiedad intelectual de los contenidos creados por el usuario}

Es habitual que, a la vez que se reconoce la propiedad intelectual de los contenidos creados por el usuario a este último, se incluyan en las cláusulas contractuales cesiones de estos derechos en favor de la plataforma. Estas licencias, al igual que sucede conotros contratos de servicios digitales, suelen ser muy amplias tanto en su ámbito territorial y temporal (ámbito territorial mundial, por tiempo indefinido o por el máximo que permita la legislación sobre propiedad intelectual aplicable al contrato) como por los derechos de explotación que se conceden (derecho de transformación, comunicación pública, distribución, etc.). Las finalidades de estas cesiones no siempre aparecen delimitadas en las condiciones predispuestas por el proveedor, ni siempre se permite al usuario oponerse a ellas.

Por otra parte, el proveedor de la plataforma suele exonerarse de responder por contenidos sujetos a derechos de propiedad intelectual de terceros publicados por los usuarios $^{25}$. Por ello, es recomendable, a nuestro parecer, que el pequeño empresario que

24 Para un estudio más detallado sobre la validez de las limitaciones y exclusiones de responsabilidad y la aplicación extensiva de la normativa de protección del consumidor a pequeños empresarios que utilicen servicios digitales, nos remitimos a ROSSELLÓ RUBERT, Francisca María, Cloud Computing. Régimen jurídico para empresarios. $1^{a}$ edición, Navarra: Thomson Reuters Aranzadi. Colección Grandes Tratados, 2018, págs. 290 a 320.

25 Las condiciones de la plataforma Minube establecen que "en caso de que el usuario integre obras de terceros, o su imagen personal, declara que cuenta con las autorizaciones correspondientes, debiendo asumir de forma exclusiva las responsabilidades que se deriven de posibles reclamaciones. La cesión antes señalada es independiente de si efectivamente se recibió contraprestación. La baja del usuario no afecta en modo alguno los términos de la cesión, por lo que si ésta ocurre, los contenidos aportados no serán retirados", y se exime de responsabilidad "Por eventuales reclamaciones de terceros por concepto de derechos de propiedad intelectual y/o patentes o marcas, en relación con cualquier tipo de contenidos que los usuarios suban al sitioMinube. Este tipo de reclamaciones será directamente derivada al usuario, el que responderá en forma íntegra por los daños, perjuicios y gastos jurídicos que se ocasionen". TripAdvisor, por su parte, advierte al usuario: "Tenga en cuenta que al enviar contenido al presente sitio web por correo electrónico, mediante publicaciones en el presente sitio web o de cualquier otro modo, incluidos todo tipo de comentarios, opiniones de hoteles, preguntas, sugerencias, fotografías o vídeos, ideas o similares que se incorporen en cualquier envío (...), usted otorga a TripAdvisor y a sus empresas afiliadas un derecho no exclusivo, sin regalías, perpetuo, transferible, irrevocable y con pleno derecho de sublicencia para (a) utilizar, reproducir, modificar, adaptar, traducir, distribuir, publicar, crear trabajos derivados, mostrar públicamente y realizar tales envíos en todo el mundo y en cualquier medio, ya sea conocido ahora o concebido a partir de este momento, para cualquier propósito; y (b) utilizar el nombre que envíe en relación con dicho envío". En nuestra opinión, esta licencia de derechos en favor de TripAdvisor resulta 
utilice estas Webs 2.0 esté informado de a existencia y contenido de estas cláusulas, y que el juzgador determine su validez en aquellos casos en los que sean susceptibles de considerarse desproporcionadas o injustificadas ${ }^{26}$.

\subsection{Limitaciones y exclusiones de responsabilidad}

Al igual que sucede con otros contratos de servicios digitales, en los contratos de plataformas comparadoras de ofertas turísticas el proveedor realiza ciertas exclusiones de responsabilidad. Entre estas exoneraciones, las más comunes son aquellas relacionadas con problemas de disponibilidad, fallos o errores de la plataforma o del software o hardware que la sustenta, con perturbaciones provocadas por terceros, con conflictos entre usuarios de la plataforma a causa de los contenidos publicados, con contenidos $\mathrm{u}$ opiniones de usuarios o con el borrado incidental de contenidos, entre otras.

A diferencia de lo que sucede en la prestación de servicios en la nube, en la que generalmente existe una relación clara entre prestador y cliente, deberemos averiguar el servicio que presta efectivamente el operador de la plataforma Web 2.0. Así, en algunas ocasiones, este operador actúa como mediador entre el empresario turístico y su potencial clientela, posibilitando que el internauta tenga conocimiento de ofertas y pueda ponerse en contacto con el proveedor turístico para contratar los servicios. Por ello, es razonable que en estos casos el proveedor se exonere de las eventualidades que puedan surgir en la relación jurídica que nazca entre el empresario turístico y el usuario de la plataforma cuando se formalice el contrato entre ambos ${ }^{27}$.

desproporcionada y desequilibrada, teniendo en cuenta la relación jurídica que vincula a plataforma y usuario. [Fecha de consulta: 24 d septiembre de 2018].

26Para un estudio más detallado de los datos digitales almacenados remotamente, nos remitimos a ROSSELLÓ RUBERT, Francisca María, Cloud Computing. Régimen jurídico para empresarios, op. cit., págs. 169 y ss.

27Como ejemplo, el aviso legal del buscador de servicios y productos Rastreator.com aclara en términos sencillos en qué consiste la actividad que presta a los usuarios de su Web 2.0, y distingue su actividad de intermediación de la actividad empresarial de las empresas cuyos precios, opiniones y servicios se comparan: "El usuario, cuando accede a obtener una comparativa de precios o tarifas de productos y servicios ofrecidos por Rastreator.com, debe recordar que Rastreator.com actúa como simple intermediario independiente a través del proveedor del servicio o producto ofertado en la website de Rastreator.com. La función de Rastreator.com se limita a ofrecer la comparativa de precios o tarifas del producto o servicio que solicite el usuario y que resulte de los datos suministrados por el usuario a través del cuestionario de Rastreator.com, (...) En esos supuestos, el usuario podrá averiguar fácilmente quién es ese proveedor, dado que la información contenida en esa website irá acompañada de un "poweredby" seguido del nombre comercial del proveedor (...). No obstante, en estos casos el proveedor se identificará al comienzo de esas comunicaciones comerciales con su nombre comercial, facilitará sus datos de contacto, al igual que hará referencia a cualquier otro dato que resulte obligatorio por Ley. (...). Rastreator.com advierte a sus usuarios que las descripciones que se hagan de los productos o servicios ofertados en la website de Rastreator.com se realizan a los meros efectos informativos, de acuerdo con la información y documentación que proporcionen a Rastreator.com los proveedores de esos productos o servicios". Al quedar fuera de la relación jurídica entre proveedor y cliente, el comparador intermediario también se exime de responsabilidades derivadas del contrato entre aquellos: "El usuario no contrata ningún servicio o producto con Rastreator.com, por lo que Rastreator.com no será responsable de las condiciones y términos de los contratos que el usuario suscriba con el proveedor correspondiente, tras la comparativa realizada. Asimismo, Rastreator.com no 
Sin embargo, existen plataformas que permiten al internauta contratar directamente los servicios turísticos, y cuya actuación es más similar a la de una agencia online de viajes (Online Travel Agency u OTA), dado que sus funcionalidades permiten introducir datos del cliente para efectuar una "reserva" del establecimiento a través del propio operador y enviar correos electrónicos como confirmación de la reserva, los cuales sirven a modo de prueba por el consumidor a la hora de efectuar el check-in en el establecimiento, o su mediación en cancelaciones de reservas. En la práctica, algunos de estos operadores manifiestan asimismo realizar únicamente actividades de intermediación, y se exoneran de cualquier responsabilidad, con lo cual no siempre aparecerá claramente delimitada contractualmente la posición del operador de la plataforma, ya que a menudo manifiesta su ajenidad a la relación entre internauta y empresario turístico. Por ello, consideramos que debe atenderse a la verdadera actividad realizada por la plataforma, (intermediación o comercialización) y no a la que afirma realizar, recogida en los acuerdos que ella misma predispone, especialmente en lo relativo a valorar la validez de la exoneración y limitación de sus responsabilidades ${ }^{28}$.

es responsable de las posibles contingencias o perjuicios que puedan surgir en la relación entre el usuario y el proveedor durante la vigencia del contrato, e incluso cuando éste haya ya finalizado". [Fecha de consulta: 24 de septiembre de 2018].

28La sentencia del Juzgado de lo Mercantil núm. 3 de Asturias, de fecha 30 de abril de 2012, determinó que la actuación del comparador Booking en su relación con el usuario que utilizaba estala plataforma para efectuar una reserva en un establecimiento hotelero iba más allá de la actuación de un mero intermediario, en su fundamento tercero, que "A juicio de este juzgador la intervención de la mercantil demandada va más allá que la mera intermediación, puesto que, en primer lugar, si su actuación se limitara a mediar entre el prestador del servicio (hotel) y el consumidor, su influencia concluiría facilitando los "links" o datos necesarios para identificar los establecimientos hoteleros, siendo absolutamente impropio o innecesario exigir que el consumidor contrate (léase "realice la reserva") a través de su propia página web) y, sobre todo, que la demandada le remita un correo electrónico de confirmación de una reserva "ajena"; (...), también carece de lógica que se registre una reserva por Booking.com, con toda la información detallada de la misma, dado que, si se trata de un tercero ajeno a la relación contractual, debería ser la prestadora del servicio hotelero quien realizara la reserva y remitiera un correo electrónico confirmatorio de la misma. (...) Otro dato significativo es el grado de intervención que tiene Booking.com en la cancelación de la reserva. (...). Parece evidente que la reserva la efectúo la compañía demandada, así como también gestionó su cancelación, y lo hizo conforme a las condiciones pactadas con su cliente (el actor). La nula intervención (aparición) del hotel en las necesarias e imprescindibles gestiones de reserva y cancelación, obligan a concluir que el vínculo jurídico surgió entre el consumidor (actor) y el prestador de servicios de reservas online de hoteles (demandada). (...) Finalmente, la propia relación existente entre Booking.com y los hoteles contempla situaciones como la presente, en la que la reclamación la realiza el cliente (consumidor) frente a la demandada. Así, en el documento 3 de los aportados junto con la contestación se incluye expresamente "la obligación que tiene en hotel de indemnizar, compensar y eximir a Booking.com de responsabilidades por deudas, costas, casos (incluyendo tarifas y gastos de abogados), daños, pérdidas, obligaciones, demandas de todo tipo, intereses, multas y procesos, que Booking.com haya pagado en los siguientes casos: (i) reclamaciones de los clientes relativas a información incorrecta, errónea o engañosa del Hotel en la Página Web (cláusula 6.2 de las condiciones de entrega de Booking.com con los establecimientos hoteleros)". Esta cláusula del contrato tipo permite apoyar la anterior conclusión: la parte contratante es Booking.com, por más que la prestadora del servicio sea un tercero ajeno (el hotel), sin perjuicio de las eventuales acciones de repetición contractual que la demandada pueda tener frente al prestador del servicio de alojamiento". Así, se declara en esta sentencia la nulidad de las cláusulas del comparador Booking.com por exclusión de responsabilidad cuando realmente se entiende que es parte contractual y no una simple intermediaria: "la demandada deberá de responder de cualquier falta de información, información inexacta, en relación con los servicios prestados o productos ofertados por el hotel, por ser este el objeto principal del contrato de reserva de hotel por ella gestionado, sin que pueda oponer frente al demandante la existencia de estas 
Por otra parte, las plataformas Web 2.0 que proporcionan datos al internauta sobre servicios proporcionados por terceros empresarios turísticos tampoco suelen responsabilizarse por las inexactitudes en esa información, generalmente facilitada por los propios empresarios turísticos, y que generalmente versa sobre precios o disponibilidad. A nuestro parecer, estas cláusulas son razonables, siempre y cuando no eximan a la plataforma de su propio deber de diligencia, y cuando sus funciones se restrinjan a las de un simple intermediario ${ }^{29}$.

Lo anterior no evita que las Webs 2.0 tengan como parte de su deber de diligencia la adopción de medidas de seguridad que garanticen la seguridad de los datos alojados en sus sistemas y la continuidad de la prestación, si bien, como sucede en otros servicios prestados en entornos digitales, las garantías que puedan ofrecerse en relación con la seguridad no sean totales ${ }^{30}$.

\section{PROBLEMAS ESPECÍfICOS ENTRE LAS PLATAFORMAS WEB $2.0 \mathrm{Y}$ EL PEQUEÑO EMPRESARIO TURÍSTICO}

Para el pequeño empresario turístico, además de lo anterior, se derivan de las plataformas comparadoras de servicios turísticos ciertas cuestiones igualmente problemáticas, de las cuales creemos conveniente destacar las consecuencias negativas de las opiniones oreviews de clientes en el prestigio de la empresa turística y la imposibilidad por parte de una empresa de eliminar cualquier referencia a su establecimiento cuando no tiene ningún vínculo jurídico con la plataforma.

cláusulas generales por su carácter abusivo. Como anticipamos, es la demandada la que entablaba una relación contractual con el consumidor por medio de la reserva, y se vincula con ella garantizando que los servicios contratados se ajustan a los términos en que fueron ofertados en su página web. Por ello, las limitaciones de responsabilidad que deriven de un incumplimiento contractual o cumplimiento defectuoso deben ser consideradas nulas por abusivas, puesto que no resulta equitativo excluir las eventuales acciones indemnizatorias o de responsabilidad contractual que pudieran corresponder a una de las partes contratantes (en este caso al cliente/consumidor), reenviando a éste a una reclamación frente a un tercero (hotel) con el que no ha establecido directamente vinculo contractual". Más información disponible en: <http://www.antunaabogados.es/documentos/BOOKING.pdf>. [Fecha de consulta: 24 de septiembre de 2018].

29 El comparador Rastreator.com recoge esta exoneración de responsabilidad en sus condiciones: "Rastreator.com trata de garantizar el mejor precio actualizado del producto o servicio cuya comparativa solicita el usuario. Sin embargo, Rastreator.com no será responsable de posibles errores u omisiones en el precio, cuando éste no coincida con el que se oferte en el enlace facilitado desde la website de Rastreator.com, que redirecciona directamente a la website del proveedor del producto o servicio elegido, siempre que la causa del error no se deba a circunstancias imputables a Rastreator.com". [Fecha de consulta: 24 de septiembre de 2018].

30Como referencia a la obligación de mantener ciertas medidas de seguridad para garantizar la prestación del servicio, sirva de ejemplo la anterior afirmación del comparador Rastreator.com: "A pesar de las medidas de seguridad que se adoptan, Rastreator.com no garantiza la ausencia de virus u otros elementos que pudieran dañar o alterar el sistema informático del usuario, ni asume Rastreator.com responsabilidad alguna derivada de ello". [Fecha de consulta: 24 de septiembre de 2018]. 


\subsection{La reputación corporativa online}

En las condiciones de plataformas Web 2.0 aceptadas por los usuarios, que tienen como funcionalidad la posibilidad de publicar opiniones o reviews sobre establecimientos $\mathrm{u}$ otros servicios turísticos de los cuales han podido disfrutar, el usuario generalmente se compromete a proporcionar únicamente datos veraces, exactos y actualizados ${ }^{31}$. Por su parte, los proveedores de estas plataformas suelen incluir en sus términos de servicio exenciones de responsabilidad respecto de la veracidad de las opiniones vertidas por los usuarios $^{32}$. Asimismo, es habitual que estos operadores se reserven el derecho de eliminar contenidos publicados por los usuarios a su entera discreción ${ }^{33}$. Si bien los operadores no se comprometen a ejercer un control exhaustivo sobre todos y cada uno de los contenidos vertidos en su plataforma, es habitual que realicen operaciones de rastreo y filtrado de opiniones cuestionables ${ }^{34}$.

Respecto de las opiniones fraudulentas, es decir, aquellas vertidas por usuarios que no han sido clientes del servicio sobre el cual opinan, o los intentos de propietarios o empleados de un establecimiento o servicio que pretenden mejorar la reputación de ese u otro establecimiento emitiendo opiniones negativas, o cualesquiera otras opiniones o comentarios que sean engañosos, las plataformas suelen utilizar también diferentes mecanismos para detectarlas ${ }^{35}$. La plataforma digital está obligada a retirar los contenidos

31 Como ejemplo, una cláusula de las condiciones de uso de la plataforma Minube: "El usuario declara y garantiza la veracidad y exactitud de los datos proporcionados a Minube y se compromete a mantenerlos actualizados en todo momento". Además, "Minube se reserva el derecho a poder eliminar sin previo aviso a usuarios registrados que no respondan a los principios de calidad y veracidad exigidos". [Fecha de consulta: 24 de septiembre de 2018].

32Minube establece que no se hará responsable "por falta de veracidad o precisión en la información, así como cambios o modificaciones que las líneas aéreas, hoteles o empresas abocadas al alquiler de coches, puedan cometer o realizar. En este sentido, se informa al usuario que Minube presta un servicio de intermediación entre el usuario y las empresas asociadas, por lo que cualquier decisión que éstas adopten en relación con disponibilidad, cambios de precios, o días y horarios, pertenece al exclusivo ámbito de su responsabilidad". [Fecha de consulta: 24 de septiembre de 2018].

33TripAdvisor "se reserva el derecho de eliminar, a su entera discreción y sin previo aviso, los mensajes de los usuarios y/o el contenido del sitio por cualquier motivo". Disponible en: <https://tripadvisor.mediaroom.com/ES-terms-of-use>. [Fecha de consulta: 24 de septiembre de 2018].

34TripAdvisor, en la información de su sitio web sobre la moderación de opiniones, afirma utilizar herramientas automáticas y equipos de moderadores que examinan opiniones cuestionables, y permiten a la comunidad de usuarios "denunciar" contenidos inapropiados de otros usuarios que sean susceptibles de infringir las Políticas de Uso Adecuado del servicio y las normas de publicación de opiniones. Disponible en: <https://www.tripadvisor.es/TripAdvisorInsights/abouttripadvisorreviews>. $\quad$ [Fecha de consulta: 24 de septiembre de 2018].

35TripAdvisor responde a la pregunta “¿Cómo determina TripAdvisor que una opinión es parcial?" distinguiendo entre opiniones pagadas por un negocio u "opiniones de alquiler", opiniones incentivadas, chantajes de clientes a establecimientos bajo amenaza de opiniones negativas, opiniones creadas por familiares, empleados o amigos del establecimiento y opiniones perjudiciales generadas por establecimientos de la competencia. Tripdavisor afirma utilizar sistemas automáticos de seguimiento y control de opiniones, afirmando la imposibilidad de revisar mediante el factor humano la cantidad ingente de opiniones que el sitio web recibe por minuto. Asimismo, publica un video del "recorrido" que realiza una opinión antes de publicarse, y de cómo es filtrada para garantizar al máximo su imparcialidad, que sean adecuadas a todos los públicos, que se basen en experiencias recientes, relevantes, etc. Aquellas que infringen claramente alguna directriz de la política de uso 
si conoce su ilicitud, como exige el artículo 16 de la LSSI, con lo cual es altamente recomendable que el pequeño empresario turístico que pueda verse perjudicado, informe de inmediato a la plataforma sobre la falsedad del contenido, aportando el máximo de pruebas que soporten su defensa y demuestren la lesión de sus derechos, para que esta retire los contenidos $^{36}$. La legislación y la jurisprudencia inicialmente consideraban responsable al autor de los comentarios, exonerando a las plataformas Web 2.0 que permitían como funcionalidad la publicación de opiniones. Sin embargo, parece que los órganos judiciales cada vez toman mayor consciencia del peso real de estas plataformas en la comercialización y promoción de servicios turísticos, y ya se toman en consideración los controles implementados por tales plataformas sobre las opiniones que publican y acogen en su Web 2.0, así como la posibilidad de sancionar a empresas o intermediarios que se dedican a comerciar con opiniones manipuladas o tendenciosas ${ }^{37}$.

El pequeño empresario turístico que se vea perjudicado frente a estas situaciones, puede iniciar acciones en defensa del derecho al honor de la empresa ${ }^{38}$ (Ley Orgánica 1/1982 de 5 de mayo, de Protección Civil del Derecho al Honor; Ley Orgánica 2/1984, sobre el derecho de rectificación; Ley 34/2002 de 11 de julio, de Servicios de la Sociedad de la Información y del Comercio Electrónico, y artículos 205 a 216 del Código Penal), con el fin de retirar los contenidos de la Red que perjudiquen su reputación. A pesar de lo

adecuado son rechazadas y se impide su publicación en la Web 2.0. Otras opiniones "sospechosas" son estudiadas más a fondo, para averiguar si se trata de opiniones incentivadas, vandálicas, o fruto de actividades comerciales de optimización de opiniones, mediante el uso de técnicas informáticas similares a las utilizadas por entidades bancarias. Una vez detectadas estas opiniones, se analizan por personas físicas expertas y se determina su veracidad o parcialidad. Este procedimiento también se realiza a instancia de los propios usuarios de la Web 2.0. que denuncian opiniones susceptibles de considerarse fraudulentas. Disponible en: https://www.tripadvisor.es/TripAdvisorInsights/w3683. [Fecha de consulta: 24 de septiembre de 2018].

36 Art. 16 LSSI: "Los prestadores de un servicio de intermediación consistente en albergar datos proporcionados por el destinatario de este servicio no serán responsables por la información almacenada a petición del destinatario, siempre que: a) No tengan conocimiento efectivo de que la actividad o la información almacenada es ilícita o de quelesiona bienes o derechos de un tercero susceptibles de indemnización, o b) Si lo tienen, actúen con diligencia para retirar los datos o hacer imposible el acceso a ellos."

37Cabe recordar que, en 2014, TripAdvisor fue sancionada en Italia con el pago de 500.000 euros por afirmar en su sitio web que las opiniones publicadas en su web eran "auténticas y genuinas", cuando su servicio alojaba opiniones engañosas y no establecía controles rigurosos de control de veracidad de la información publicada por usuarios. Para un estudio más detallado, nos remitimos a MARTíNEZ NADAL, Apol-lònia, ROSSELLÓ RUBERT, Francisca María, "La experiencia del cliente: análisis jurídico de la publicación online de comentarios de clientes. En particular, el caso de TripAdvisor", Actas de las IX Jornadas de Investigación en Turismo, Sevilla, 2016, págs. 99 a 128. Por otro lado, en la web de TripAdvisor se expone un caso detectado de negocio ilegal en Italia, en el cual una empresa ofrecía servicios de manipulación fraudulenta de opiniones para mejorar la valoración de establecimientos turísticos. Disponible en: https://www.tripadvisor.es/TripAdvisorInsights/w3683. [Fecha de consulta: 24 de septiembre de 2018]. La sentencia de este caso tuvo lugar el pasado 12 de septiembre de 2018 en Italia, condenándose al propietario de la empresa de comercialización de opiniones PromoSalento a nueve meses de prisión y a una indemnización de 8.000 euros.

38 Del cual es titular, como reconoció el Tribunal Constitucional en su Sentencia 139/1995. Al respecto, ver MARTÍNEZ NADAL, Apol·lònia, ROSSELLÓ RUBERT, Francisca María, La experiencia ..., op. cit, pág. 105. 
anterior, en nuestra opinión, el establecimiento debe igualmente plantearse la idoneidad de ejercer acciones legales a través de la vía judicial, debido a múltiples circunstancias, como la necesidad de aportar pruebas que acrediten los hechos concurridos o su falsedad, la imputabilidad de estos hechos a una persona física (muchas veces las opiniones se dan de manera anónima), la determinación del perjuicio al establecimiento, los largos trámites y plazos que supone un procedimiento judicial, etc ${ }^{39}$.

Por otra parte, los términos de uso de estas plataformas suelen establecer las consecuencias de la detección de estas opiniones engañosas, aunque estas consecuencias serán determinadas a discreción del proveedor ${ }^{40}$. Conscientes de esta problemática, también se han desarrollado iniciativas en el sector turístico para promover prácticas más equitativas, que destacan los puntos clave. Recordemos que su valor jurídico no es vinculante, sino que tiene los efectos de simples recomendaciones ${ }^{41}$.

\subsection{La salida de la empresa turística de la plataforma Web 2.0}

Una de las cuestiones que se plantea el pequeño empresario del sector turístico es cómo salir de una plataforma que aloja opiniones o reviews, en la cual nunca se ha dado de alta ni comparte vínculo contractual alguno (incluso, puede ocurrir que esta empresa turística ni siquiera tenga página web propia o perfil público en ninguna red social). En este caso, han sido terceros quienes, al verter sus opiniones sobre el servicio turístico y en uso de las funcionalidades de esa plataforma, involucran al pequeño empresario en la comunidad de usuarios, sin que este, como titular del establecimiento o proveedor del

39 En todo caso, puede ser desaconsejable para el pequeño empresario entrar en discusiones con usuarios descontentos utilizando como vía la misma plataforma de opiniones. Como muestra, el caso de la chef mallorquina Macarena de Castro, quien contestó a una crítica negativa a su menú, en el cual se lo consideraba una "estafa", afirmando que los clientes fueron irrespetuosos e intentaron sustraer del restaurante un elemento decorativo. En la sentencia, dictada por el Juzgado de Primera Instancia número 5 de Inca ( y por tanto, recurrible), la chef fue condenada a una sanción de 6.000 euros, ya que en el contenido de la respuesta, la chef hizo públicos, a través del sistema de comentarios de la propia plataforma, datos referentes a la privacidad, honor e intimidad de los clientes de su restaurante, como su nombre y profesión, o el hecho de afirmar que presentaban signos de embriaguez durante su visita al establecimiento hostelero galardonado con una estrella Michelín.

40Estas son las consecuencias de TripAdvisor ante la detección de opiniones fraudulentas: "Se producen varias consecuencias si determinamos que se han enviado opiniones fraudulentas a un establecimiento: El establecimiento puede caer varias páginas en el índice de popularidad de TripAdvisor. Los datos de clasificación de los establecimientos se gestionan caso por caso y son de nuestra propiedad. El establecimiento perderá su derecho a participar en los premios TravellersChoice de TripAdvisor, en las listas Top 10, en las notas de prensa, etc. Aparecerá un mensaje de penalización, rojo y grande, en la página del perfil del establecimiento, indicando que las opiniones del establecimiento son sospechosas".

41Destacaremos la aprobación de las directrices "BenchmarksofFairPractices in Online Distribution", por parte de la Asociación Empresarial Europea de Hoteles, Restaurantes y Cafeterías (HOTREC), en las que se dedica un apartado a las opiniones online de clientes sobre estas categorías de establecimientos. El apartado 17 destaca las cuestiones más importantes para una valoración justa y equitativa de los servicios prestados por el sector hostelero, como la falta de control editorial, la falta de herramientas que detecten opiniones manipuladas, la posibilidad de un derecho de réplica, etc. HOTREC, "BenchmarksofFairPractices in Online Distribution" [en línea]. Disponibles en: <http://www.hotrec.eu>. [Fecha de consulta: 24 de septiembre de 2018]. Ver también MARTÍNEZ NADAL, Apol·lònia, ROSSELLÓ RUBERT, Francisca María, La experiencia..., op. cit, pág. 116. 
servicio turístico valorado, haya tenido la posibilidad de consentir o negarse.

La eliminación de contenidos de la plataforma queda a discreción del propio proveedor, tal y como suele establecer en sus términos de uso. Ello, no obstante, perjudica al pequeño empresario turístico, al no poder decidir libremente sobre si quiere o no que su establecimiento aparezca en la concreta Web 2.0, independientemente de la existencia o no de opiniones negativas de usuarios sobre la calidad de su servicio o establecimiento.

Los proveedores establecen que, al tratarse de comunidades de usuarios, se busca la obtención de la máxima información sobre la calidad de cualesquiera establecimientos turísticos de esa categoría que oferten servicios en el mercado. Algunos operadores manifiestan que su plataforma también sirve para "denunciar" servicios deficientes o prestados por empresas que han intentado falsear de algún modo su reputación online, con lo cual, para que el consumidor turístico esté alertado si decide contratar en este tipo de establecimientos, no solo no les permiten retirar su presencia del sitio web, sino que etiquetan al establecimiento a modo de advertencia ${ }^{42}$.

Si bien estamos a favor de que el consumidor tenga una opinión libre y pueda expresarla y compartirla en comunidad, también consideramos que deben ofrecerse mecanismos de defensa a las empresas perjudicadas por opiniones de usuarios. Debe tenerse en cuenta que la empresa turística, aunque puede ver perjudicada su reputación y menguados sus ingresos con ciertas opiniones vertidas en Webs 2.0 que permitan la expresión de opiniones de usuarios, no siempre tiene ocasión de alegar o defenderse. Existen muchas circunstancias que no pueden valorarse con una simple opinión de un cliente, como sucesos ocasionados de manera puntual, mal interpretados, manipulados o falsos, que tuvieron lugar años atrás, que sucedieron bajo las directivas de otros propietarios o gestores, etc.

Además, aunque la plataforma decida atender a la petición de retirar las referencias sobre una empresa o de opiniones sobre esta vertidas por usuarios, generalmente no se establece un procedimiento transparente o rápido para ello, con lo cual la opinión o la empresa sigue estando accesible para el resto de la comunidad de viajeros durante un tiempo, con los consiguientes perjuicios para la reputación y el lucro cesante que ello supone. Tampoco se asegura que, tras finalizar este proceso de retirada de la opinión u opiniones por parte de la plataforma, esta comunique a la comunidad de internautas tal rectificación, o muestre un mensaje que permita limpiar la reputación dañada de la empresa que no lo merecía, a modo de "opinión falsa", "incidencia solventada", "cambios

\footnotetext{
${ }^{42}$ A falta de relación contractual entre plataforma web y establecimiento hotelero, debería darse la posibilidad al empresario hostelero de poder desaparecer de los establecimientos valorados. A nuestro parecer, debería existir un equivalente al "derecho al olvido" cuyos titulares fuesen empresas y otras personas jurídicas, cuyos representantes legales deben poder optar y tomas decisiones libremente que afecten a su reputación online y a las estrategias de promoción de la marca comercial que representan. En la red ya existen propuestas independientes de reclamaciones que los empresarios pueden dirigir a las plataformas para reclamar la baja de sus establecimientos. A modo de ejemplo, la propuesta del abogado de Galicia Tito Petrizzo en su blog https://www.modelosycontratos.com/2016/02/como-eliminar-negocio-tripadvisor/.
} 
en la dirección del establecimiento", etc.

Para solventar estas cuestiones, proponemos al legislador una regulación similar al "derecho al olvido" que tenga como destinatarias a las personas jurídicas, y especialmente al pequeño empresario turístico, para que pueda decidir libremente sobre si quiere o no pertenecer a este tipo de plataformas y para que pueda defenderse de potenciales abusos, y no quede desamparado ante actuaciones arbitrarias de clientes u operadores de Webs 2.0. Es razonable entender que los representantes legales de un negocio o empresa (socios, propietarios, gestores, etc.) deben disponer de mecanismos de defensa y maniobra para planificar y mejorar la reputación online de su establecimiento o marca comercial, sin que se les impida por parte de una plataforma la baja de las opiniones referentes a su establecimiento, con la cual, además, no media acuerdo contractual alguno. Asimismo, hay que tener en consideración que la reputación de un establecimiento puede evolucionar, por ejemplo, haciendo mejoras en la propiedad o dirección del establecimiento, en su gestión, en la plantilla o en las instalaciones, etc., con lo cual las opiniones previas a esas mejoras que no hayan sido identificadas o eliminadas pueden distorsionar la competitividad del establecimiento.

Asimismo, es acertado, en nuestra opinión, que ya existan sanciones para ciertas prácticas comerciales engañosas, como la publicación de opiniones falsas o manipuladas sobre establecimientos que se promocionan a través de plataformas de opinión, independientemente de que las vierta el propio establecimiento $\mathrm{u}$ otro establecimiento de la competencia o un usuario interesado. No obstante, también deberá ponderarse este derecho del empresario a retirar su presencia de ciertas plataformas con otros derechos de terceros, como puede ser el derecho a la libertad de expresión del usuario y a la libertad de información.

\section{CONCLUSIONES}

Al compartir la problemática común de la contratación de servicios digitales (cumplimiento de políticas de uso adecuado, propiedad intelectual, limitaciones y exclusiones de responsabilidades, etc.), el pequeño empresario turístico se enfrenta apotenciales situaciones de desequilibrio contractual y abuso por parte del proveedor digital, que es quien predispone las cláusulas de un contrato en línea no negociable. En estos casos, y siempre que su situación de desamparo sea asimilable a la del consumidor, puede apelar a la aplicación extensiva de la normativa de protección al consumidor, tanto genérica como especial para consumo de servicios digitales.

En cuanto a las plataformas comparadoras de precios y publicación de opiniones, estas pueden suponer distintos problemas al pequeño empresario del sector turístico, especialmente las referentes a los efectos legales de opiniones vertidas por otros internautas y sus consecuencias en la reputación empresarial, así como su imposibilidad práctica de dar de baja a su establecimiento de plataformas. En este último caso, puesto 
que no ha existido ningún tipo de contrato ni consentimiento previo para que el establecimiento aparezca en la plataforma, consideramos que sería idónea la regulación de un derecho para empresarios equivalente al derecho al olvido de las personas físicas, que permita salvaguardar la reputación de un determinado comercio o establecimiento y orientar su promoción de acuerdo con las decisiones del propietario o del equipo encargado de su gestión.

\section{BIBLIOGRAFÍA}

APARICIO VAQUERO, Juan Pablo, Negocios y contratación en Internet. El ejemplo de los portales de ocio y viajes. En: BUENO DE MATA; Federico (coord.), FODERTICS, Estudios sobre Derecho y Nuevas Tecnologías. La Coruña: Andavira Editora, 2012, págs. 53-78.

AGENCIA ESPAÑOLA DE PROTECCIÓN DE DATOS (AEPD), Plan de inspección de oficio a cadenas hoteleras, 2004.Disponible en:https://www.agpd.es/portalwebAGPD/canaldocumentacion/recomendaciones/common/pdf S/RECOMENDACIONESCADENASHOTELERASDEFINITIVAS.pdf

BATUECAS, Alfredo; APARICIO, Juan P., La contratación online de servicios turísticos. En: PANIZA, Antonia (coord.), Nuevas fórmulas de comercialización "on line" de servicios turísticos, subsunción en los tipos legales y distribución de responsabilidad. Granada: Editoral Comares, 2013, págs. 49 a 78.

CHANDRAKUMAR, T; PARTHASARATHY, S, A frameworkforEvaluating Cloud Enterprise ResourcePlanning (ERP) Systems. En: MAHMOOD, Zaigham (ed.), ContinuedRiseoftheClouds. Advances and Trends in Cloud Computing. Londres: Springer,2014,págs. 161-175.

CLOUD COMPUTING EUROPEAN STRATEGY, Cloud ServiceLevelAgreementStandardisationGuidelines, 2013. Disponible en: https://ec.europa.eu/digital-agenda/en/news/cloud-service-level-agreement-standardisationguidelines

CLOUD STANDARDS CUSTOMER COUNCIL, Public Cloud ServiceAgreements: WhattoExpect and WhattoNegociate. Disponible en: http://www.cloudcouncil.org/PublicCloudServiceAgreements2.pdf

CLOUD STANDARDS CUSTOMER COUNCIL, PracticalGuideto Cloud ServiceAgreementsVersion 2.0, 2015. Disponible en: http://www.cloudcouncil.org/CSCC_Practical_Guide_to_Cloud_Service_Agreements_Version_2.0.pdf

DOMINGO, Miguel Á., CHÁVEZ, Esther; ESCOBAR, Bernabé, Evolución del Software y herramientas de Revenue Management utilizados por hoteles. En: Actas del XI Congreso Internacional de Turismo y Tecnologías de la Información y la Comunicación TuriTec, 2016. Málaga: UMA Universidad de Málaga, 2016, págs. 484 a 503. 
FORO TURISMO.AS;SOCIEDAD ESTATAL PARA LA GESTIÓN DE LA INNOVACIÓN Y LAS TECNOLOGÍAS TURÍSTICAS (SEGITTUR), ThinkTankCloud Computing y Turismo, 2012. Disponible en: http://www.segittur.es/opencms/export/sites/segitur/.content/galerias/descargas/notasprensa/Informe-conclusiones-Think-Tank-Cloud-Computing-y-Turismo.pdf

FUNDACIÓN TELEFÓNICA, Desarrollo Empresarial y Redes Sociales. El caso de las microempresas españolas, 2015. Disponible en: http://www.fundaciontelefonica.com/arte_cultura/publicaciones-listado/pagina-itempublicaciones/itempubli/331/

FUNDACIÓN ORANGE, España: La transformación digital en el sector turístico, 2016. Disponible en: http://www.fundacionorange.es/wpcontent/uploads/2016/05/eE_La_transformacion_digital_del_sector_turistico.pdf

GONZÁLEZ, Javier, ¿Qué significa realmente el porcentaje de Uptime?, Blogs Teoriza de Tecnología e informática, 2011. Disponible en: http://gonzo.teoriza.com/significa-realmente-porcentaje-uptime-burbuja-disponibilidad

GUASCH PORTAS, Vicent; SOLER FUENSANTA, José; Cloud Computing, turismo y protección de datos. En: Revista de Análisis Turístico, núm. 17. Málaga: UMA Universidad de Málaga, 2014, págs. 61-68.

HOSTELTUR, Priceline compra la startup española Hotel Ninjas. Disponible en: https://www.hosteltur.com/159150_priceline-compra-start-up-espanola-hotel-ninjas.html

HOTREC, BenchmarksofFairPractices in Online Distribution. Disponible en: http://www.hotrec.eu

INCIBE, Nueve claves de ciberseguridad para el sector del Turismo, 2016. Disponible en: https://www.incibe.es/protege-tu-empresa/blog/9-claves-ciberseguridad-el$\underline{\text { sector-del-turismo }}$

Disponible

INTECO, Guía para empresas: identidad digital y reputación online, 2012. http://empresasyemprendedores.aytosalamanca.es/es/downloads/guia identidad reputacion e mpresas_final_nov2012x1x.pdf

INCIBE, Riesgos y Amenazas en Cloud Computing, 2011. Disponible en http://cert.inteco.es/extfrontinteco/img/File/intecocert/EstudiosInformes/cert_inf_riesgos_y_a menazas_en_cloud_computing.pdf

Junta de Andalucía, Sociedad Andaluza para el desarrollo de las telecomunicaciones SANDETEL, Cloud Computing aplicado a los sectores de la agroindustria, eficiencia energética, industrias culturales y turismo, 2012. Disponible en: http://www.juntadeandalucia.es/export/drupaljda/analisis_cloud_computing.pdf

MARTÍNEZ NADAL, Apol-lònia, Las centrales electrónicas de reservas turísticas: breves consideraciones acerca de su naturaleza y régimen jurídico. En: TOBÍO RIBAS, Ana M, FERNÁNDEZ-ALBOR BALTAR, Ángel (coords.), Libro homenaje al Prof. Dr. José Antonio Gómez Segade: Madrid: Marcial Pons, 2013, págs. 967-984. 
MARTINEZ NADAL, Apol-lònia, Reputación online de las empresas de alojamiento turístico y publicación por terceros de informaciones negativas (reviews), En: MORILLAS María José, et. al., (dirs.), Estudios sobre el futuro Código Mercantil: libro homenaje al profesor Rafael Illescas Ortiz. Madrid: Universidad Carlos III. Disponible en: http://hdl.handle.net/10016/21115

MARTINEZ NADAL, Apol-lònia, Publicación en páginas web de valoraciones falsas con incidencia en la reputación digital de los empresarios de alojamiento ¿una práctica desleal? En: Revista de Derecho Mercantil, núm. 297, 2015, págs.139-169.

MARTÍNEZ NADAL, Apol-lònia, Las centrales electrónicas de reservas turísticas, En. TOBÍO RIBAS, Ana M. (coord.) Estudios de Derecho Mercantil. Libro homenaje al Prof. J.A. Gómez Segade. Madrid: Marcial Pons, 2013, págs. 967-984.

MARTÍNEZ NADAL, Apol-lònia, Las cláusulas de paridad en las relaciones entre OTA (Online Travel Agencies) y empresarios de alojamiento turístico: presente y futuro. En: Diario La Ley, núm. 8574, 2015, págs. 1 y ss.

MARTÍNEZ NADAL, Apol-lònia, Derecho de la competencia y cláusulas de paridad en la comercialización turística electrónica. En: MARTÍNEZ NADAL, Apol-lònia (coord.) Turismo y Nuevas Tecnologías. Navarra: Editorial Thomson Civitas, 2016, págs. 10 y ss.

MARTÍNEZ NADAL, Apol-lònia, ROSSELLÓ RUBERT, Francisca María, La experiencia del cliente: análisis jurídico de la publicación online de comentarios de clientes. En particular, el caso de TripAdvisor. En: Actas de las IX Jornadas de Investigación en Turismo, Sevilla, 2016, págs. 99 a 128.

MARTÍNEZ NADAL, Apol-lònia, ROSSELLÓ RUBERT, Francisca María, Comercio electrónico y Derecho de la Competencia: las cláusulas de paridad en el sector turístico. En: BUENO DE MATA; Federico (dir.), Hacia una justicia 2.0. Actas del XX Congreso Iberoamericano de Derecho e Informática. Salamanca: Ratio Legis, 2016, págs. 387-402.

MARTÍNEZ NADAL, Apol-lònia(coord.), Turismo y Nuevas Tecnologías, 1a edición, Navarra: Aranzadi, 2016, 209 págs.

Observatorio Nacional de las Telecomunicaciones y la Sociedad de la Información ONTSI; Entidad Pública Empresarial RED.ES. Informe Diagnóstico Tecnológico Turismo rural, 2007. Disponible en: https://es.scribd.com/document/34878355/Diagnosticotecnologico-Turismo-rural-2007

Observatorio Nacional de las Telecomunicaciones y la Sociedad de la Información ONTSI, La sociedad en Red 2015. Disponible en: http://www.ontsi.red.es/ontsi/sites/ontsi/files/Informe\%20Anual\%20La\%20Sociedad\%20en\% 20red\%202015\%20\%28Edici\%C3\%B3n\%202016\%29.pdf

Observatorio Nacional de las Telecomunicaciones y la Sociedad de la Información ONTSI,Informes e-Pyme. Año 2016. Disponible en: http://www.ontsi.red.es/ontsi/sites/ontsi/files/epyme_15_analisis_sectorial_de_implantacion_de_las_tic_en_la_pyme_espanola.pdf. Año 
2014. Disponible en: http://www.ipyme.org/Publicaciones/informe-epyme-2014.pdf. Año 2013, Disponible en: http://www.red.es/redes/sala-de-prensa/convocatorias/presentacion-del\%E2\%80\%98informe-epyme-2013-analisis-sectorial-de-implantacion

PALOS SÁNCHEZ, Pedro R.; AGUAYO CAMACHO, Mariano, Los ecosistemas de Turismo Inteligente basados en Cloud Computing. En: El Turismo y la experiencia del cliente, Actas de las IX Jornadas de Investigación en Turismo, Tomo II: Sevilla: Universidad de Sevilla, 2016, págs. 125-146.

ROSSELLÓ RUBERT, Francisca María, Cloud Computing. Régimen jurídico para empresarios. 1a edición, Navarra: Thomson Reuters Aranzadi. Colección Grandes Tratados, 2018, 448 págs.

ROSSELLÓ RUBERT, Francisca María, Las contraprestaciones no dinerarias en la Propuesta de Directiva sobre suministro de contenidos digitales. En: Revista de Derecho Mercantil, núm. 303, 2017, págs. 163-190.

SOFTDOIT, Las herramientas de marketing y ventas y el uso del CRM en las empresas españolas en 2016, 2017. Disponible en: https://www.softwaredoit.es/estudio/estudio-herramientas-marketing-ventas-uso-softwarecrm-empresas-espana-2016.html

TECNOHOTEL, Hotel Ninjas se alía con Salesforce para ampliar sus servicios en la nube. Disponible en: https://www.tecnohotelnews.com/2016/07/infografia-mas-del-50-delas-empresas-espanolas-tienen-alojadas-sus-soluciones-crm-en-la-nube 